\title{
ASPECTOS BIOESTRATIGRÁFICOS DE LOS AMMONITES E INOCERÁMIDOS (BIVALVIA) DEL ALBIENSE SUPERIOR AL MAASTRICHTIENSE DE LA PROVINCIA DE ÁLAVA
}

\author{
Robert SANTAMARÍA ${ }^{1}$ y Gregorio LÓPEZ1 \\ ' Departament de Geología. Facultat de Cièncias. Universitat Autònoma de Barcelona. 08193 Bellaterra.
}

Santamaría, R. y López, G. 1996. Aspectos bioestratigraficos de los ammonites e inoceramidos (Bivalvia) del Albiense Superior al Maastrichtiense de la provincia de Alava. [Biostratigraphic aspects of the Upper Albian to Maastrichtian ammonoids and inoceramids (Bivalvia) from the Alava province]. Revista Española de Paleontología, N Extraordinario, 148-159. ISSN 0213-6937.

\begin{abstract}
The stratigraphic distribution of Cretaceous ammonite and inoceramid faunas from Alava is discussed. Some species of ammonites and inoceramids are recognized, ranging from the Upper Albian to the Maastrichtian. Main biostratigraphic work is focused on Campanian and Maastrichtian faunas, where accurate field data is available. Many other specimens of the Museo de Ciencias Naturales de Alava don't have good stratigraphic data related, so they are only placed in the ammonite and inoceramid zonal scheme previously established (Lopez, 1992b \& 1994, Lopez \& Santamaria, 1992 and Santamaria, 1991 \& 1992). Other important taxa (e.g. some groups of bivalves, gastropods, and annelids) are also discussed, and some of them are listed for the first time in northern Spain.
\end{abstract}

Keywords: ammonites, inoceramids, biostratigraphy, Cretaceous, Navarro-Cantabrian Basin, Alava.

\section{RESUMEN}

Se discute la distribución estratigráfica de la fauna de ammonites e inoceramidos del Cretácico de la provincia de Álava. Se reconocen numerosas especies de ammonites e inoceramidos desde el Albiense superior al Maastrichtiense. Sólo se ha realizado un estudio bioestratigráfico suficientemente preciso en el Campaniense y el Maastrichtiense, pisos en los que se pudo realizar trabajo de campo y situar la fauna. Una gran parte del restante material procedente de las colecciones del museo de Ciencias Naturales de Álava no muestra un suficiente control estratigráfico, por lo que sólo ha sido correlacionado con las zonas de ammonites e inoceramidos establecidas previamente (López, 1992 b y 1994, López y Santamaría, 1992 y Santamaría, 1991 y 1992). Se reconoce la presencia de otros taxones relevantes (por ej. diversos grupos de bivalvos, gasterópodos y anélidos), y algunos se citan por primera vez en el norte de España.

Palabras clave: ammonites, inocerámidos, bioestratigrafia, Cretácico, Cuenca Navarro-Cántabra, Álava.

\section{INTRODUCCIÓN}

En las colecciones de Paleontología del Museo de Ciencias Naturales de Álava (M.C.N.A.), en Vitoria-Gasteiz, se encuentra depositada una importante representación de la rica fauna de ammonoideos e inocerámidos, asi como de otra fauna (diversos grupos de bivalvos, gasterópodos, annélidos, etc.), del Cretácico superior de Álava, junto con fauna representativa de Burgos y Navarra.

Este trabajo abarca dos objetivos. Por un lado se ha procedido a estudiar la colección de ammonites e inocerámidos del Albiense al Santoniense superior, que mayoritariamente se encontraba depositada en el M.C.N.A., que fue parcialmente completada por los autores, y que ha permitido perfeccionar las zonaciones ya existentes (López, 1992b y 1994, López y Santamaría, 1992 y Santamaría, 1991 y 1992). Por otro lado, se ha realizado un estudio bioestratigráfico detallado de diversos afloramientos del Campaniense y Maastrichtiense, habiéndose muestreado numerosas secciones, lo que permitió recolectar más ejemplares y ubicar correctamente los depositados en el
M.C.N.A. Cabe indicar que como resultado de un minucioso estudio sistemático de otros taxones, se ha reconocido un gran número de especies de grupos que generalmente no han sido citados en las publicaciones sobre el Cretácico del norte de España.

Los yacimientos estudiados en este trabajo se reparten esencialmente por la parte central y meridional de Álava y el extremo occidental de Navarra (Fig. 1). Geográficamente se encuentran situados en una posicion intermedia entre tres unidades fisiográficas bien diferenciadas: la Cordillera Cantábrica al oeste, los Pirineos al este y la Cuenca del Ebro al sur. El material procedente de Navarra corresponde a un único yacimiento, localizado en la cantera de cemento de Olazagutia, situada en la parte occidental de la provincia de Navarra, cerca de su límite con la provincia de Álava. Asimismo, se ha estudiado material procedente de localidades burgalesas, como Laño (Condado de Treviño) y Nidáguila.

En concreto, la inmensa mayoría de los ejemplares estudiados procede de los alrededores de las estribaciones montañosas situadas en la zona centro-oriental de Álava. El Puerto de Vicuña y la localidad de Andoin, situadas en la Sierra 


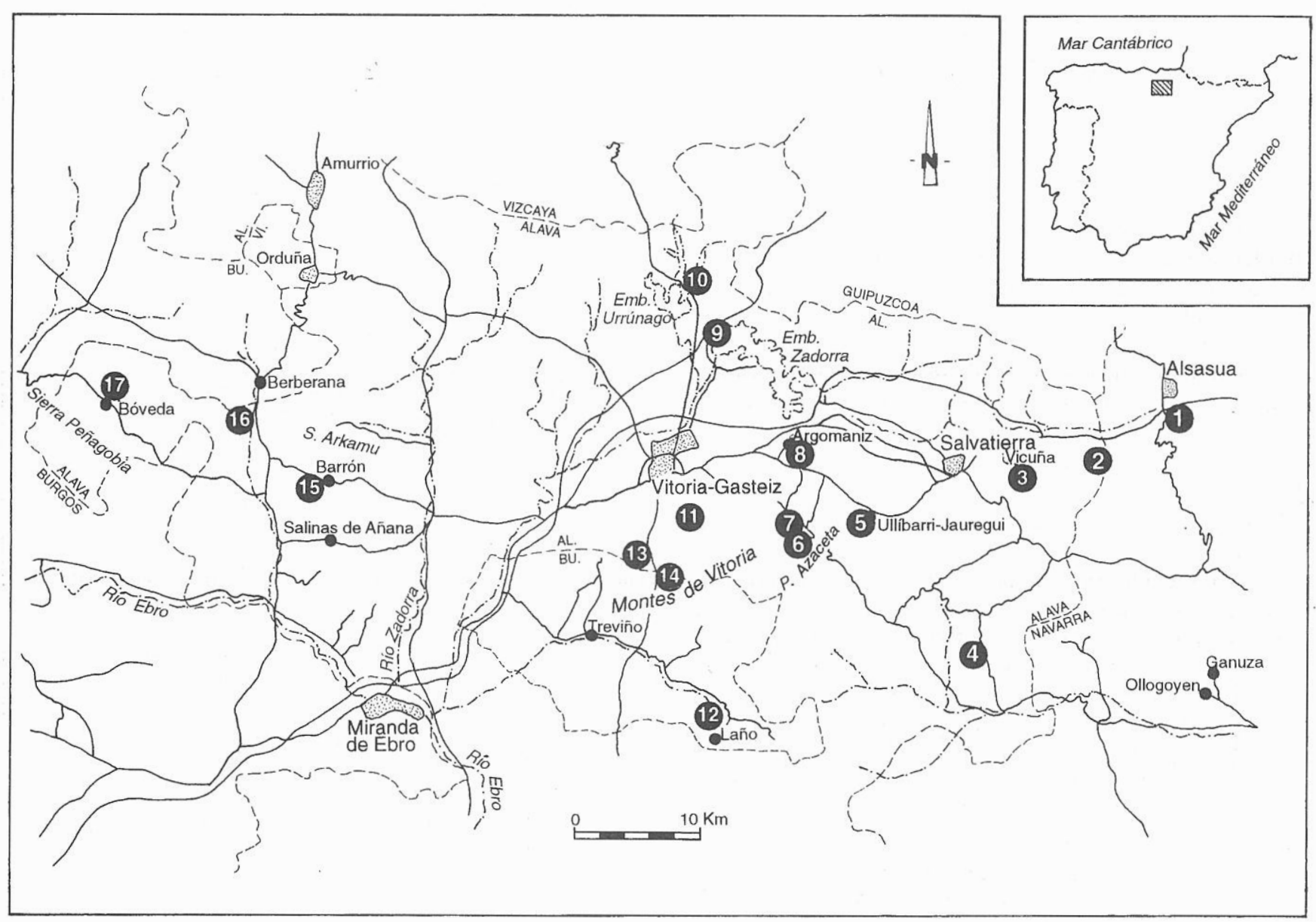

Figura 1. Situación geográfica de las series y yacimientos estudiados. 1: Olazagutía; 2: Andoin; 3: Puerto de Vicuña; 4: Oteo; 5: UllíbarriJauregui I y II; 6: Balsa del Puerto de Azaceta; 7: Eguileta I y II; 8: Argómaniz; 9: Embalses del Zadorra y Ullíbarri Gamboa; 10: Legutiano/Villareal de Álava; 11: Gámiz; 12: Laño; 13: Puerto de Vitoria I; 14: Puerto de Vitoria II; 15: Barrón norte; 16: Fresneda y 17: Bóveda, Bóveda norte y puerto de Bóveda.

de Entzia, y el Puerto de Vitoria y el yacimiento de Ullíbarri-Jauregui, situados estos dos ultimos en los Montes de Vitoria, son los yacimientos que han proporcionado un mayor numero de ejemplares. Asimismo, han proporcionado ejemplares los yacimientos de los alrededores de Villareal de Álava y de los embalses del Zadorra, al norte de Vitoria, así como los de Bóveda, Bóveda norte y puerto de Bóveda situados en la Sierra de Peñagobía.

\section{SITUACIÓN GEOLÓGICA}

Geológicamente, los yacimientos estudiados corresponden a afloramientos de Cretácico situados en la parte centro-meridional del Dominio Vasco-Cantábrico. Dentro de ésta, la mayoría se encuadran dentro de la Cuenca Navarro-Cántabra, mientras que tan sólo uno (Nidáguila) pertenece a la Plataforma Norcastellana.

Los afloramientos del Cretácico superior de la Cuenca Navarro-Cántabra fueron divididos por Amiot (1982) en siete sectores, en funcion de las diferencias que existen entre sus depósitos y su repartición geográfica. El material estudiado en este trabajo procede únicamente de tres de ellos: a) Valles de Losa y Mena. Donde se localizan los diversos yacimientos santonienses de Bóveda, dentro de la Formación Losas.

b) Cuenca de Vitoria. En este sector se localizan la mayor parte de los yacimientos estudiados, en concreto los de Legutiano/Villareal de Álava del Albiense (Fm. Valmaseda), los de Ullíbarri-Gamboa y embalses del Zadorra, del Cenomaniense (Fm. de Valmaseda y la Fm. Arceniega), los de Andoin, Ullíbarri-Jauregui y el puerto de Vitoria, del Campaniense (Fm. Vitoria), y el del puerto de Vicuña, del Maastrichtiense (Fm. Puerto de Olazagutia).

c) La Barranca. En este sector tan sólo se ha estudiado el yacimiento de Olazagutía, del Coniaciense-Santoniense, cuyos materiales corresponden a la Fm. de Olazagutía.

También se han estudiado ejemplares procedentes de la Plataforma Norcastellana, en concreto de la región septentrional o 1 de Floquet et al. (1982). Esta comprende la parte suroccidental de la Cordillera Cantábrica y el extremo suroriental de Asturias. En esta región se pueden distinguir dos partes diferenciadas, una al norte y otra al sur del Alto Ebro, siendo las relaciones con la cuenca Navarro-Cántabra más 
claras en la primera de ellas. Sin embargo, es en la parte meridional, en la que los medios de plataforma tienden a ser protegidos, en la que se ha recolectado fauna, que procede de Terradillos de Sedano y Nidáguila.

\section{ANTECEDENTES}

Los ammonoideos y bivalvos del Cretácico de la Península Ibérica han sido citados desde hace más de 150 años en algunos de los trabajos estratigráficos fundamentales de Europa occidental, entre los que cabe destacar el trabajo de d'Orbigny (1850).

El hecho de que la Península Ibérica estuviese situada durante el Cretácico entre las provincias faunísticas del Tethys y de Europa Occidental, confiere una importancia singular a su fauna de ammonideos y bivalvos, puesto que dicha posición intermedia implica que, dependiendo del momento geológico, estas faunas habían de estar influenciadas por una u otra provincia.

Los ammonites del Cretácico superior de la región han sido objeto de numerosos estudios ya desde el siglo pasado. Entre los trabajos pioneros cabe destacar los de Karrenberg (1935) y Ciry (1940), que incluían también algunos aspectos bioestratigráficos. Wiedmann (1960, 1962, 1964, 1975 y 1979) realizó una serie de trabajos paleontológicos y bioestratigráficos en la mayor parte de la cuenca, sentando la base sobre la que se desarrollaron estudios posteriores. En algunos de ellos (Santamaría, 1991 y 1992) se revisaron y actualizaron los esquemas desarrollados por Wiedmann en parte de la Cuenca Navarro-Cántabra y en la Plataforma Norcastellana.

Los inocerámidos han sido utilizados en bioestratigrafía desde hace mucho tiempo, debido a su abundancia, a su presencia en una amplia diversidad de facies y a su frecuente asociación, durante el Cretácico, con ammonites. Su gran utilidad bioestratigráfica está también en función de su amplia repartición geográfica, ya que el $75 \%$ de las especies de inocerámidos poseen una distribucion intercontinental, que excede a la de muchos otros macrofósiles cretácicos. Asimismo, parece que su dispersión ha sido geológicamente instantánea, tan rápida como la de otros grupos fósiles (López, 1990a y 1994).

El estudio de los bivalvos (excluidos los inocerámidos y rudistas) del Cretácico superior de la Península Ibérica no sólo no había sido abordado de manera sistemática, sino que ni tan siquiera se solía citar su presencia en aquellas secciones en que aparecían otros grupos (ammonites, inocerámidos, etc.). Hasta el momento, los estudios sobre bivalvos se restringían a los concernientes a grupos específicos como inocerámidos y rudistas, frente a los estudios de carácter general. De hecho, los únicos trabajos que abordaron exclusivamente el estudio de los bivalvos (excluyendo los inocerámidos y los rudistas) del Cretácico de la Península Ibérica fueron los trabajos de Dhondt (1982) y López (1993).

\section{ASOCIACIONES Y EDAD DE LAS FAUNAS}

La distribución vertical de las asociaciones faunísticas aquí estudiadas, especialmente las de ammonites (lámina I) e inocerámidos (lámina II), ha permitido en todos los casos la datación de las localidades que las contienen.

En su conjunto, el material estudiado abarca el intervalo Albiense superior-Maastrichtiense superior, y sólo en el Campaniense y el Maastrichtiense se pudo tener un adecuado control estratigráfico, que permitió el establecimiento de una zonación informal para el Campaniense superior.

\section{ALBIENSE SUPERIOR}

Localidades: Legutiano/Villareal de Álava.

Procedencia de la fauna: Colecciones del MCNA y privadas. Sin correcto control estratigráfico.

Litología: Margas areniscosas de la Fm. Valmaseda.

Asociación faunística:

Ammonites: Desmoceras (Desmoceras) latidorsatum (Michelin), Stoliczkaia (Stoliczkaia) dispar (d'Orbigny), Stoliczkaia (S.) sp., Mortoniceras (Mortoniceras?) nanum Spath, Scaphites (Scaphites) hugardianus (d'Orbigny), Anisoceras pseudoelegans (Pictet y Campiche), A. arrogans (Giebel), A. perarmatum (Pictet y Campiche), A. saussureanum (Pictet), Anisoceras sp., Idiohamites sp., Pseudohelicoceras? sp., Mariella (Mariella) bergeri (Brogniart), Mariella (M.) quadrituberculata (Bayle), Mariella (M.) sp. y Lechites gaudini (Pictet y Campiche).

Inocerámidos: Inoceramus athabaskensis McLearn.

Bioestratigrafía: La fauna de ammonites de estas dos localidades indica en su conjunto una edad de Albiense superior (Fig. 2), con especies típicas de la Zona de Stolicz-

\begin{tabular}{|c|c|c|}
\hline Cenomaniense superior & $\begin{array}{l}\text { Loc.: Embalses del Zadorra. } \\
\text { Fm. Arceniega. }\end{array}$ & Euomphaloceras euomphalum (Sharpc) \\
\hline Cenomaniense medio & $\begin{array}{l}\text { Loc.: Embalses del Zadorra } \\
\text { y Ullibarri Gamboa. } \\
\text { Fm. Arceniega. }\end{array}$ & 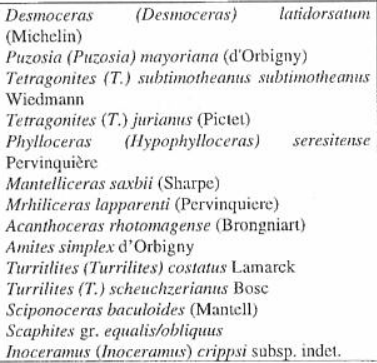 \\
\hline Cenomaniense inferior & $\begin{array}{l}\text { Loc.: Embalses del Zadorra. } \\
\text { Fm. Arceniega. }\end{array}$ & $\begin{array}{l}\text { Mantelliceras saxbii (Sharpc) } \\
\text { Mrhiliceras lapparenti (Pervinquiere) }\end{array}$ \\
\hline Albiense superior & $\begin{array}{l}\text { Loc.: Legutiano y Villareal } \\
\text { dc Alava. } \\
\text { Fm. Valmaseda. }\end{array}$ & $\begin{array}{l}\text { Desmoceras (Desmoceras) latidorsatum } \\
\text { (Michelin) } \\
\text { Stoliczkaia (Stoliczkaia) dispar (d'Orbigny) } \\
\text { Stoliczkaia (S.) sp. } \\
\text { Mortoniceras (Mortoniceras?) nanum Spath } \\
\text { Moroniceras durnovarites perinflatum (Spath) } \\
\text { Scaphites (Scaphites) hugardianus (d'Orbigny) } \\
\text { Anisoceras pseudoelegans (Pictet y Campiche) } \\
\text { A. arrogans (Giebel) } \\
\text { A. perarmatum (Pictet y Campiche) } \\
\text { Anisoceras sp. } \\
\text { Pseudohelicoceras? sp. } \\
\text { Mariellla (Mariella) bergeri (Brogniart) } \\
\text { Mariellla (M.) quadrituberculata (Bayle) } \\
\text { Mariella (M.) sp. } \\
\text { Lechites gaudini (Pictet y Campiche) } \\
\text { Inoceramus athabaskensis McLearn }\end{array}$ \\
\hline
\end{tabular}

Figura 2. Listado de los ammonites e inocerámidos del Albiense superior al Cenomaniense superior de la provincia de Álava, con indicación de las localidades y formaciones litoestratigráficas en que ha sido reconocido dicha fauna. 
kaia dispar sin diferenciar. Esta zona es hoy en día equivalente al sub-piso Vraconiense, que ha quedado en desuso (Hancock, 1991).

Dentro de esta zona se reconocen dos subzonas, que varían segun la región. En el noroeste de Europa (zonación estándar) se reconoce la Subzona de Mortoniceras (Mortoniceras) rostratum (inferior) y la de Mortoniceras (Durnovarites) perinflatum (superior). Equivalentes a éstas son, según Kennedy (1985), las subzonas del norte de España definidas por Wiedmann (1979): Pervinqueria fallax (inferior) y Paraturrilites (Bergericeras) quadrituberculatus (= Mariella (Mariella) quadrituberculata) (superior). En este trabajo se ha identificado Mortoniceras (D.) perinflatum (localidad de Zubielqui, Navarra) y Mariella (M.) quadrituberculata (pantano de Ordunte), por lo que al menos estas dos localidades pueden ser datadas con mas precisión dentro de la Subzona de $M$. (D.) perinflatum.

Por lo que se refiere a la fauna de inocerámidos, tan sólo se han estudiado en detalle ejemplares de Inoceramus athabaskensis. Sin embargo, existe un gran número de ejemplares de esta especie y otras (por ej. Inoceramus dunvaganensis McLearn) en la colección privada de Don Francisco Ignacio Vaz, que todavía no ha sido estudiada adecuadamente. Dicha fauna tiene una gran importancia, que viene dada por varias razones:

a) Es la primera cita de estas especies en territorio europeo, ya que hasta el momento su registro se restringía al Norte de los Estados Unidos y a Canadá (Mc Learn, 1943 y Kauffman, 1977).

b) Su posición estratigráfica es coincidente con la de los ammonites citados anteriormente.

c) Es bastante probable la presencia de algunas especies aún no descritas.

d) Su presencia en regiones europeas puede aclarar la evolución de varios grupos filogenéticos de inocerámidos.

\section{CENOMANIENSE}

Localidades: Embalses del Zadorra y Ullíbarri Gamboa.

Procedencia de la fauna: Colección del MCNA y privadas. Sin correcto control estratigráfico. niega.

Litología: Margas y calizas arcillosas de la Fm. Arce-

\section{Asociación faunística:}

Ammonites: Desmoceras (Desmoceras) latidorsatum (Michelin), Puzosia (Puzosia) mayoriana (d'Orbigny), Tetragonites (Tetragonites) subtimotheanus subtimotheanus Wiedmann, T. jurianus (Pictet), Phylloceras (Hypophylloceras) seresitense Pervinquiere, Mantelliceras saxbii (Sharpe), Acanthoceras rhotomagense (Brongiart), Euomphaloceras euomphalum (Sharpe), Mrhiliceras lapparenti (Pervinquiere), Hamites simplex d'Orbigny, Turrilites (Turrilites) costatus Lamarck, Turrilites (T.) scheuchzerianus Bosc, Sciponoceras baculoides (Mantell) y Scaphites gr. equalis/obliquus. indet.

Inocerámidos: Inoceramus (Inoceramus) crippsi subsp.

Otros taxones relevantes: Codakia (Epilucina) sp. y Anadara sp.

Bioestratigrafía: Esta asociación (Fig. 2), de la que se carece como en el caso anterior de un adecuado control estra- tigráfico, está constituida por especies que abarcan en su conjunto el intervalo Cenomaniense inferior-parte baja del Cenomaniense superior, si bien de una manera discontinua. La parte baja del Cenomaniense inferior se reconoce por la presencia de Mantelliceras saxbii y Mrhiliceras lapparenti, mientras que la parte baja del superior lo hace por la aparicion de Euomphaloceras euomphalum. El resto de especies indicaría en su conjunto una edad de Cenomaniense medio, parte inferior.

Inoceramus (Inoceramus) crippsi es una especie bien conocida en el Cenomaniese medio europeo.

\section{CONIACIENSE SUPERIOR}

Localidad: Olazagutía

Procedencia de la fauna: Colección del MCNA. Sin correcto control estratigráfico.

Litología: Calizas arcillosas de la Fm. de Olazagutía.

Asociación faunística:

Inocerámidos: Magadiceramus subquadratus subquadratus (Schluter) y Magadiceramus subquadratus subsp. indet.

Bioestratigrafía: Esta asociación (Fig. 3) corresponde al Coniaciense superior, y se reconoce por la aparición de cualquiera de las especies de Magadiceramus, en este caso

\begin{tabular}{|c|c|c|}
\hline $\begin{array}{l}\text { Campaniense superior } \\
\text { Asociación II }\end{array}$ & $\begin{array}{l}\text { Loc.: Puerto de Vitoria II. } \\
\text { Fm. Vitoria. }\end{array}$ & $\begin{array}{l}\text { Trachyscaphites spinigeri porschi (Adkins) } \\
\text { Pachydiscus (Pachydiscus) haldemsis (Schlüter) } \\
\text { Pachydiscus (P.) sp. } \\
\text { Phylloceras (Hypophylloceras) sp. } \\
\text { Psendophyllites sp. } \\
\text { Baculites spp. }\end{array}$ \\
\hline $\begin{array}{c}\text { Campaniense superior } \\
\text { Asociación I }\end{array}$ & 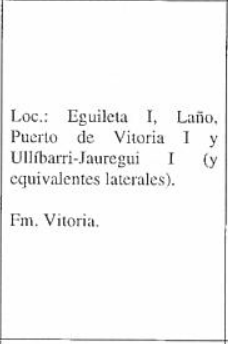 & $\begin{array}{l}\text { Hoplitoplacenticeras marroti (Coquand) } \\
\text { Menabites (Bererella?) sp. } \\
\text { Scaphites (Scaphites) gibbus Schlüter } \\
\text { Pseudoxybeloceras (Parasolenoceras) phaleratum } \\
\text { (Griepenkerl) } \\
\text { Baculites spp. } \\
\text { Platyceramus adjakendsis (Aliev) } \\
\text { Endocostea balticus haldemensis (Giers) } \\
\text { Endocostea ef. pterioides (Giers) } \\
\text { Selenoceramus decipiens (Zittel) } \\
\text { Selenoceramus n. sp. aff. inflexus ((Beyenburg) } \\
\text { Selenoceramus cf. n. sp. aff. decipiens (Zittel) } \\
\text { Cataceramus sagensis nebrascensis (Meek) } \\
\text { Cataceramus n. sp. 1 } \\
\text { Cataceramus cf. buguntaensis (Dobrov y } \\
\text { Pavlova) }\end{array}$ \\
\hline Campaniense inferior & $\begin{array}{l}\text { Loc.: Oteo y Gámiz, } \\
\text { Fm. Vitoria. }\end{array}$ & $\begin{array}{l}\text { Pachydiscus (Pachydisctus) launayi (de } \\
\text { Grossouvre) } \\
\text { Endocostea balticus marcki (Giers) } \\
\text { Cataceramus } \mathrm{n} \text {. sp. } 4\end{array}$ \\
\hline $\begin{array}{l}\text { Santonicnse sin } \\
\text { diferenciar }\end{array}$ & $\begin{array}{l}\text { Loc.: Bóveda y Fresneda. } \\
\text { Fm. Losas. }\end{array}$ & $\begin{array}{l}\text { Placenticeras polyopsis (Dujardin) } \\
\text { Texanites (Texanites) gallicus Collignon } \\
\text { Texanites (T,) hispanicus Collignon } \\
\text { Eulophoceras nov. sp. } \\
\text { Baculites incurvatus (Dujardin) }\end{array}$ \\
\hline Santoniense superior & $\begin{array}{l}\text { Loc.: Argomaniz, Boveda y } \\
\text { Olazagutía. } \\
\text { Fm. de Olazagutí. } \\
\text { Fm. Losas. }\end{array}$ & $\begin{array}{l}\text { Platyceramus cycloides ricardoi (López) } \\
\text { Platyceramus cycloides alssenensis (Seitz) } \\
\text { Cordiceramus muelleri muelleri (Petrascheck) } \\
\text { Cordiceramus cf. muelleri (Pctrascheck) } \\
\text { Cordiceramus brancoiformis (Seitz) } \\
\text { Endocostea balticus balticus (Böhm) } \\
\text { Selenoceramus sp. }\end{array}$ \\
\hline $\begin{array}{c}\text { Santoniense } \\
\text { medio-superior }\end{array}$ & $\begin{array}{l}\text { Loc.: Barrón norte. } \\
\text { Fm. Losas. }\end{array}$ & $\begin{array}{l}\text { Platyceramus cycloides cycloides (Wegner) } \\
\text { Platyceramus cycloides subsp. indet. } \\
\text { Cordiceramus bueltenensis cf. bueltenensis (Seitz) } \\
\text { Cordiceramus bueltenensis amoldi (Seitz) }\end{array}$ \\
\hline Santoniense inferior & $\begin{array}{l}\text { Loc.: Olazagutía. } \\
\text { Fm. de Olazagutía }\end{array}$ & Platyceramus undulatoplicatus michaeli (Heinz) \\
\hline Coniaciense superior & $\begin{array}{l}\text { Loc:: Olazagutía. } \\
\text { Fm. de Olazagutía. }\end{array}$ & $\begin{array}{l}\text { Magadiceramus subquadratus subquadratus } \\
\text { (Schlüter) } \\
\text { Magadiceramus subquadratus subsp. indet. }\end{array}$ \\
\hline
\end{tabular}

Figura 3. Listado de los ammonites e inocerámidos del Coniaciense superior al Campaniense superior (Asociación II) de la provincia de Álava, con indicación de las localidades y formaciones litoestratigráficas en que ha sido reconocida dicha fauna. 
por la presencia de subespecies de Magadiceramus subquadratus. Esta asociación ya fue reconocida por López (1992b y 1994) en la Cuenca Navarro-Cántabra, aunque anteriormente este género ya había sido utilizado por Wiedmann y Kauffman (1978) en su tentativa de subdividir el Coniaciense. Ello coincide asimismo, con los datos proporcionados por Sornay (1983 y 1984) en su subdivisión del Coniaciense del Sureste de Francia y por López (1986) en su subdivisión de la región Surpirenaica Central.

Segun Tröger (1989), la parte basal del Coniaciense superior está caracterizada principalmente por Magadiceramus subquadratus Schluter, incluyendo inocerámidos involutos y Sphenoceramus? subcardissoides Heinz (especie de la Zona 23 de Tröger). En la parte superior del Coniaciense superior (Zona 24 de Tröger) los inocerámidos involutos ya no están presentes. Por ello, y como ya indicó López (1992) esta asociación en la Cuenca Navarro-Cántabra corresponde al Coniaciense superior, y es equivalente a las Zonas 23 y 24 de Tröger (1989). Puesto que en el Coniaciense de la Cuenca Navarro-Cántabra no se ha identificado ningún ejemplar de Volviceramus, no se puede diferenciar la Zona 23 de la Zona 24, tal como fueron establecidas por Tröger (1989).

\section{SANTONIENSE INFERIOR}

Localidad: Olazagutía

Procedencia de la fauna: Colección del MCNA. Sin correcto control estratigráfico.

Litología: Calizas arcillosas de la Fm. de Olazagutía.

Asociación faunística: (Heinz).

Inocerámidos: Platyceramus undulatoplicatus michaeli

Bioestratigrafía: Esta asociación (Fig. 3) corresponde al Santoniense inferior y es equivalente a la Zona 26 de Tröger (1989) y a la Zona de Undulatoplicatus de Seitz (1961). En la Cuenca Navarro-Cántabra, la base del Santoniense está bien definido por la primera aparición de cualquiera de las subespecies de Platyceramus undulatoplicatus Romer (López, 1992b y 1994). De estas subespecies, la más frecuente es Pl. undulatoplicatus michaeli, que se puede localizar por toda la Cuenca Navarro-Cántabra, como ya indica López (1992). De acuerdo con Sornay (1978, 1983 y 1984) la base del Santoniense en las distintas cuencas de Francia también se reconoce por las primeras apariciones de Platyceramus undulatoplicatus, al igual que en la región Surpirenaica Central (López, 1986).

La Zona 25 de Tröger (1989) no se puede distinguir en la Cuenca Navarro-Cántabra, ya que está definida en base a la primera aparición de Sphenoceramus pachti pachti Archangelsky y subespecies, y este género no está presente en el Norte de España.

\section{SANTONIENSE MEDIO-SUPERIOR}

Localidad: Barrón norte.

Procedencia de la fauna: Recolectada por los autores.

Litología: Alternancia de margas y calizas margosas de la Fm. Losas.

Asociación faunística:
Inocerámidos: Platyceramus cycloides cycloides (Wegner), Platyceramus cycloides subsp. indet., Cordiceramus bueltenensis cf. bueltenensis (Seitz) y Cordiceramus bueltenensis arnoldi (Seitz).

Bioestratigrafía: Las primeras apariciones de las subespecies de Cordiceramus bueltenensis se registran en el Santoniense medio, como ya indican Seitz (1965) y López (1992b). De todas formas, su distribución estratigráfica puede alcanzar la base del Santoniense superior.

\section{SANTONIENSE SUPERIOR}

Localidades: Argomaniz, Bóveda y Olazagutía.

Procedencia de la fauna: Colección del MCNA y privadas. Sin correcto control estratigráfico.

Litología: Calizas arcillosas de la Fm. de Olazagutía y una alternancia de margas y calizas margosas de la Fm. Losas.

Asociación faunística:

Inocerámidos: Platyceramus cycloides ricardoi (López), Platyceramus cycloides ahsenensis (Seitz), Cordiceramus mиelleri muelleri (Petrascheck), Cordiceramus cf. muelleri (Petrascheck), Cordiceramus brancoiformis (Seitz), Endocostea balticus balticus (Böhm) y Selenoceramus sp.

Otros taxones relevantes: Spondylus (Spondylus) spinosus (Sowerby), Pterotrigonia (Pterotrigonia) sp. 2, Plagiostoma hoperi Mantell, Pterotrigonia (Pterotrigonia) sp. 1, Amphidonte (Ceratostreon) pliciferum (Dujardin), Pycnodonte (Phygraea) vesiculare (Lamarck) y Poromya frequens?

Bioestratigrafía: Según la zonacion de Seitz (1965) para el Santoniense del Norte de Alemania, la Zona Muelleri corresponde a la parte inferior del Santoniense.superior, y se define por la primera aparicion de Cordiceramus muelleri. Igualmente, Sornay (1984 y 1983) considera que la base del Santoniense superior del Sureste de Francia está caracterizada por la presencia de Cordiceramus muelleri. De todas formas, puesto que no se conocen con exactitud los niveles de los que proceden los ejemplares de Cordiceramus muelleri estudiados en este trabajo, no es factible subdividir el Santoniense superior.

Por otra parte, López (1992b y 1994) indica que el Santoniense superior de la Cuenca Navarro-Cántabra se reconoce por las primeras apariciones de Cordiceramus brancoiformis, mientras que la parte terminal del Santoniense, y que con ciertas dudas podría corresponder ya al Campaniense basal, esta caracterizada por Platyceramus cycloides ricardoi, como también sugiere Kauffman (1987, comunicación personal).

La Zona 29 de Tröger (1989) no puede ser reconocida en el Norte de España, ya que considera que la asociación característica está formada principalmente por Sphenoceramus patootensiformis, Sphe. angustus Beyenburg, así como por pequeños inocerámidos semejantes a Sphe. lingua Goldfuss; pero el género Sphenoceramus no está presente en el norte de la Península Ibérica.

Por su parte, la subespecie Platyceramus cycloides ahsenensis (Seitz) ha sido reconocida por López (1992b y 1994) en toda la Cuenca Navarro-Cántabra, y su registro estratigráfico abarca a todo el Santoniense, al igual que en gran parte de Europa. 


\section{SANTONIENSE SIN DIFERENCIAR}

Localidades: Bóveda y Fresneda.

Procedencia de la fauna: Colección del MCNA, privadas y recolectada por los autores. Lamolda y Martínez (1986), López (1992a) y Santamaría (1991 y 1992) publicaron la serie de Fresneda en la que se ha situado la fauna.

Litología: Alternancia de margas y calizas margosas de la Fm. Losas.

Asociación faunística:

Ammonites: Placenticeras polyopsis (Dujardin), Texanites (Texanites) gallicus Collignon, Texanites (T.) hispanicus Collignon, Eulophoceras nov. sp. y Baculites incurvatus (Dujardin).

Otros taxones relevantes: Modiolus cf. capitatus (Zittel) y Neithea (Neithea) regularis (Schlotheim).

Bioestratigrafía: Estas y otras localidades coetáneas han sido ya estudiadas por Santamaría (1991 y 1992), e indican una clara edad Santoniense, Zona de Placenticeras polyopsis. Al igual que Kennedy (1987) en el área tipo, Santamaria fue incapaz de subdividir el Santoniense en más de una zona, y utilizó la misma zonación de Kennedy. Sin embargo, Hancock (1991), subdivide el Santoniense del área tipo en dos zonas, la de Texanites gallicus (inferior) y la de Placenticeras paraplanum (superior). Esto es muy distinto a lo que sucede con los inocerámidos, que sí permiten subdividirlo (Fig. 3).

\section{CAMPANIENSE INFERIOR}

Localidades: Oteo y Gámiz.

Procedencia de la fauna: Colección del MCNA, privadas y recolectada por los autores.

Litología: Margas y calizas arcillosas de la Fm. Vitoria. Asociación faunística:

Ammonites: Pachydiscus (Pachydiscus) launayi (de Grossouvre).

Inocerámidos: Endocostea balticus marcki (Giers) y Cataceramus n. sp. 4

Bioestratigrafía: Si bien la evidencia es escasa, la especie Pachydiscus (Pachydiscus) launayi parece ser una buena indicadora del Campaniense inferior. Así sucede en Aquitania, donde aparece asociado a Placenticeras bidorsatum, y en Madagascar (Kennedy, 1986). En unos niveles aparentemente más altos que los de Gámiz se recolectó también un fragmento de Baculites sp.

La subespecie Endocostea balticus marcki suele ser común en el Campaniense inferior de Europa (Tröger, 1989), aunque también aparece citada en el Campaniense superior; en este último caso suelen corresponder a citas en trabajos sin un buen control estratigráfico, por lo que provisionalmente la consideramos con reservas como del Campaniense inferior (Fig. 3).

\section{CAMPANIENSE SUPERIOR}

Esta edad ha resultado la más propicia para el estudio bioestratigráfico, con ammonites, debido a la relativa abundancia y variedad de faunas (Figs. 3 y 4), muchas ellas bien conocidas en todo el mundo, y al control estratigráfico deta-

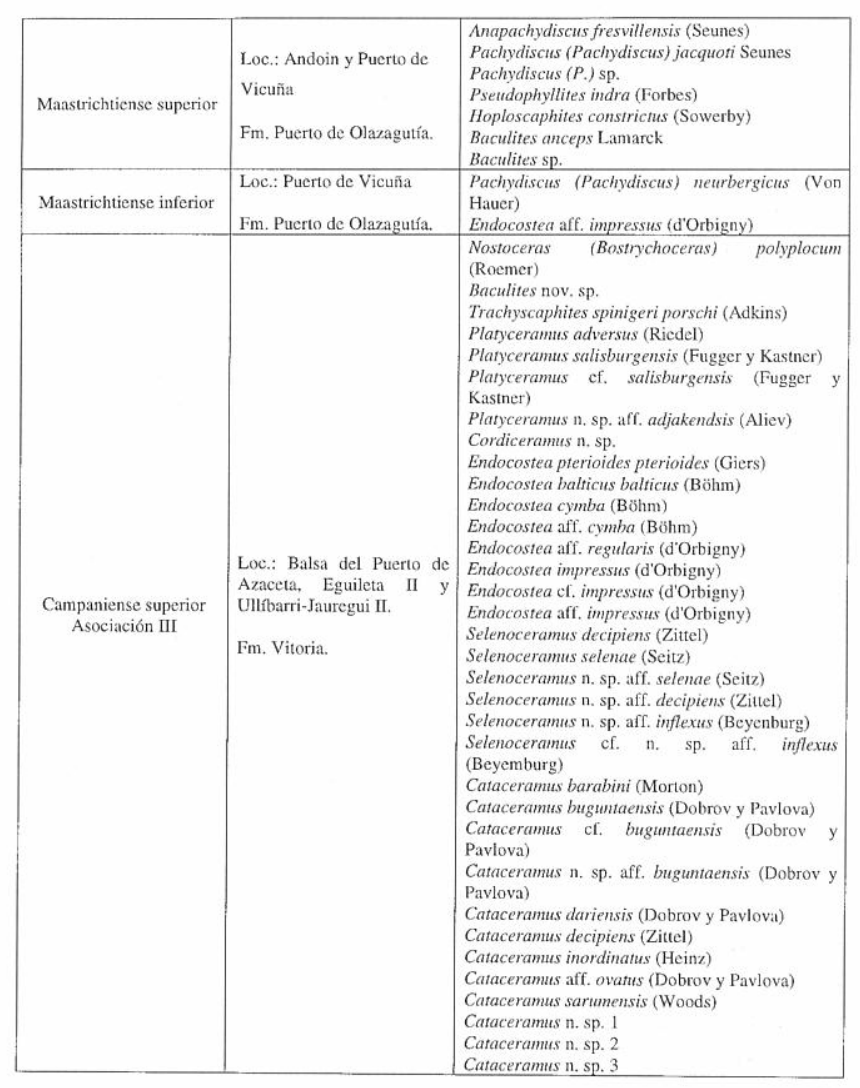

Figura 4. Listado de los ammonïtes e inocerámidos del Campaniense superior (Asociación III) al Maastrichtiense superior de la provincia de Álava, con indicación de las localidades y formaciones litoestratigráficas en que ha sido reconocida dicha fauna.

llado que se pudo llevar a cabo, pudiéndose determinar con gran certeza la procedencia de la mayoría del material depositado en el Museo de Ciencias Naturales de Álava.

Cabe decir que, por lo que respecta a los inocerámidos, no existe una subdivisión del Campaniense superior suficientemente valida. La más reciente tentativa corresponde al trabajo de Tröger (1989), pero en él todavía existen algunas lagunas (López, en prensa).

\section{Asociación I}

Localidades: Eguileta I, Laño, Puerto de Vitoria I (Cerro testigo) y Ullíbarri-Jauregui I (y equivalentes laterales).

Procedencia de la fauna: Colección del MCNA y privadas, junto a fauna recolectada por los autores. Se realizó la serie de Ullíbarri-Jauregui I (López y Santamaría, 1994 y Santamaría, en prensa).

Litología: Margas y calizas arcillosas de la Fm. Vitoria. Asociación faunística:

Ammonites: Hoplitoplacenticeras marroti (Coquand), Menabites (Bererella?) sp., Scaphites (Scaphites) gibbus Schlüter, Pseudoxybeloceras (Parasolenoceras) phaleratum (Griepenkerl) y Baculites spp.

Inocerámidos: Platyceramus adjakendsis (Aliev), Endocostea balticus haldemensis (Giers), Endocostea cf. pterioides (Giers), Selenoceramus decipiens (Zittel), Selenocera- 
mus n. sp. aff. inflexus (Beyenburg), Selenoceramus cf. n. sp. aff. decipiens (Zittel), Cataceramus sagensis nebrascensis (Meek), Cataceramus n. sp. 1 y Cataceramus cf. buguntaensis (Dobrov y Pavlova).

Otros taxones relevantes: Spondylus (Spondylus) cf. spinosus (Sowerby), Spondylus aff. latus (Sowerby), Pterotrigonia (Scabrotrigonia) cf. vaelsensis (Böhm), Lyropecten (Aequipecten) aff. campaniensis (d'Orbigny), Amphydonte (Ceratostreon) pliciferum (Dujardin), Hyostissa ? semiplana (Sowerby), Pycnodonte (Phygraea) vesiculare (Lamarck) y Pycnodonte (Phygraea) aff. vesiculare (Lamarck).

Bioestratigrafía: Esta asociación (Fig. 3) puede considerarse, al menos parcialmente, equivalente a la zona de Hoplitoplacenticeras marroti, que normalmente marca la base del Campaniense superior. Sin embargo, éste es un tema sujeto a intensas discusiones en este momento, y recientemente Hancock (1991) senala que la Zona de H. marroti quedaria separada de la base del Campaniense superior por un "gap" o hiatus estratigráfico, cosa que sucede tanto en el norte de Europa como en Madagascar.

En la parte oriental de La Barranca, Küchler y Kutz (1989), reconocen una zona marroti/O. pilula (un equínido), para la base del Campaniense superior. Ésta equivaldría al tercio inferior de la zona estandar marroti, que quedaría completada con otras dos zonas de equínidos (E. subglobosa y $E$. conica) y previas a la aparición de Bostrychoceras polyplocum. Resulta difícil por el momento asegurar si esta zona con $H$. marroti y la asociación aquí definida son totalmente equivalentes.

Otras especies interesantes que aparecen en Ullíbarri-Jauregui son Parasolenoceras phaleratum, que en Polonia es índice de la parte mas baja del Campaniense superior y Scaphites gibbus, asociada con $H$. marroti en el área tipo, pero común en la parte alta del Campaniense inferior del occidente de Alemania.

Se ha correlacionado la fauna piritizada de Baculites de Eguileta I con la de la localidad de Puerto de Vitoria I (Cerro testigo), esta última portadora únicamente de $H$. marroti. Estos baculítidos parecen además ser idénticos a los asociados con H. marroti en Ullíbarri-Jauregui.

\section{Asociación II}

Localidad: Puerto de Vitoria II.

Procedencia de la fauna: Colección del MCNA y privadas, junto a fauna recolectada por los autores. Se realizó la serie de Pto. Vitoria (López y Santamaría, 1994 y Santamaría, en prensa).

Litología: Margas y calizas arcillosas de la Fm. Vitoria. Asociación faunística:

Ammonites: Trachyscaphites spinigeri porschi (Adkins), Pachydiscus (Pachydiscus) haldemsis (Schluter), Pachydiscus (P.) sp., Phylloceras (Hypophylloceras) sp., Pseudophyllites sp. y Baculites spp.

Otros taxones relevantes: Neithea (Neithea) sexcostata (Woodward), Spondylus (Spondylus) truncatus (Lamarck), Granocardium cf. noeggerathi (Müller), Amphydonte (Ceratostreon) sp., Exogyra (Exogyra) overwegi Buch, Corbicula sp., Granocardium sp., Panopea cf. gurgitis (Brongniart), Venericor planicosta? y Owenirhynchia aff. rubra (Calzada).
Bioestratigrafia: En la serie de Puerto de Vitoria, Carmelo Corral nos informó que los niveles portadores de esta asociación son más altos que otros que contendrían una fauna de baculítidos piritizados, que lamentablemente no se han podido estudiar por el momento. Luego, si asumimos que todos los niveles con ammonites piritizados (Eguileta I, Puerto de Vitoria I (Cerro testigo) y éste de los baculítidos) son equivalentes, entonces los niveles piritizados que menciona Corral deberían pertenecer a la Asociación I, y por tanto ser ésta más vieja que los niveles con la Asociación II, que los suprayace.

Esto parece respaldado por la ausencia de $H$. marroti, así como la presencia de Pachydiscus haldemsis. Hancock (1991) senala que $P$. haldemsis esta presente en la zona de Bostrychoceras polyplocum, pero que su rango probablemente sea diferente al de la especie índice. En Polonia $P$. koenei $(=P$. haldemsis) se encuentra tanto en las zonas de Pseudoxybeloceras phaleratus como la de B. polyplocum. En Urdiroz/Imiscoz (Navarra), Küchler y Kutz (1989) reconocen una zona local de $P$. haldemsis/E. conoidea, parcialmente equivalente a la zona de E. cónica en la Barranca oriental, que como ya se mencionó antes es suprayacente a la zona de H. marroti. Esto parece indicar que en esta región $P$. haldemsis está situado claramente por encima de H. marroti y por debajo de B. polyplocum.

\section{Asociación III}

Localidades: Balsa del Puerto de Azaceta, Eguileta II y Ullíbarri-Jauregui II.

Procedencia de la fauna: Colección del MCNA y privadas, junto a fauna recolectada por los autores. Se realizó la serie de Ullíbarri-Jauregui II (López y Santamaría, 1994 y Santamaría, en prensa).

Litología: Margas y calizas arcillosas de la Fm. Vitoria. Asociación faunística:

Ammonites: Nostoceras (Bostrychoceras) polyplocum (Roemer), Baculites nov. sp. y Trachyscaphites spinigeri porschi (Adkins).

Inocerámidos: Platyceramus adversus (Riedel), Platyceramus salisburgensis (Fugger y Kastner), Platyceramus cf. salisburgensis (Fugger y Kastner), Platyceramus n. sp. aff. adjakendsis (Aliev), Cordiceramus n. sp., Endocostea pterioides pterioides (Giers), Endocostea balticus balticus (Böhm), Endocostea cymba (Böhm), Endocostea aff. cymba (Böhm), Endocostea aff. regularis (d'Orbigny), Endocostea impressus (d'Orbigny), Endocostea cf. impressus (d'Orbigny), Endocostea aff. impressus (d'Orbigny), Selenoceramus decipiens (Zittel), Selenoceramus selenae (Seitz), Selenoceramus n. sp. aff. selenae (Seitz), Selenoceramus n. sp. aff. decipiens (Zittel), Selenoceramus n. sp. aff. inflexus (Beyenburg), Selenoceramus cf. n. sp. aff. inflexus (Beyemburg), Cataceramus barabini (Morton), Cataceramus buguntaensis (Dobrov y Pavlova), Cataceramus cf. buguntaensis (Dobrov y Pavlova), Cataceramus n. sp. aff. buguntaensis (Dobrov y Pavlova), Cataceramus dariensis (Dobrov y Pavlova), Cataceramus decipiens (Zittel), Cataceramus inordinatus (Heinz), Cataceramus aff. ovatus (Dobrov y Pavlova), Cataceramus sarumensis (Woods), Cataceramus n. sp. 1, Cataceramus n. sp. 2 y Cataceramus n. sp. 3.

Otros taxones relevantes: Arca aquisgranensis (Müller), Chlamys cf. faujasi (Defrance), Lima cf. canalifera Goldfuss, 
Modiolus (Modiolus) capitatus (Zittel), Neithea (Neithea) striatocostata (Goldfuss), Ostrea (Ostrea) sp., Panopea gurgitis (Brongniart), Pinna (Pinna?) quadrangularis Goldfuss, Plagiostoma hoperi Mantell, Proveniella sp., Pycnodonte (Phygraea) aff. vesiculare (Lamarck), Rastellum diluvianum (Linnaeus), Scaphella sp., Syncyclonema nilssoni (Goldfuss), Volutilithes sp., Vultogryphaea aff. vultur (Coquand), Vultogryphaea? sp., Weyla (Weyla) n. sp. aff. alatus (Buch), Owenirhynchia cf. globata (Arnaud), Glomerula cf. gordialis (Schlotheim), Parsimonia aff. parsimonia Regenhardt y Parsimonia sp.

Bioestratigrafía: La presencia de $N$. (B.) polyplocum permite correlacionar estos niveles con la zona de la que es índice, ampliamente reconocida en casi todo el mundo. Trachyscaphites spinigeri es una especie que en Aquitania aparece en la zona de $H$. marroti, que en el oeste de Alemania aparece por debajo de $N$. (B.) polyplocum, y que en Polonia es común a las zonas de $P$. phaleratum y $N$. (B.) polyplocum. Esto muestra un amplio rango de aparición, que podría incluir practicamente todo el Campaniense superior salvo la parte más basal. La subespecie $T$. spinigeri porschi, que es la aquí encontrada, únicamente se conocía en Texas y en Israel.

Por lo que respecta a los inocerámidos, y como ya se ha comentado anteriormente, no se puede establecer una subdivisión del Campaniense superior, puesto que la mayoría de especies han sido creadas en base a material de Europa oriental y su control estratigráfico era muy pobre. De todas formas, se puede observar que varias especies de inocerámidos coexisten en los mismos niveles que los ammonites, lo cual permitirá precisar la edad de varias de las especies de inocerámidos, especialmente de aquellas que son nuevas (Fig. 4). Por tanto, este trabajo puede ser la base de una futura subdivisión del Campaniense superior en base a inocerámidos, que vendría complementado por los datos de López, Küchler y Tröger (1992) sobre la cercana Barranca (López, en prensa).

\section{MAASTRICHTIENSE INFERIOR}

Localidad: Puerto de Vicuña

Procedencia de la fauna: Colección del MCNA. Se realizó la serie de Pto. de Vicuña (López y Santamaría, 1994).

Litología: Margas arenosas y calizas bioclásticas Fm. del Puerto de Olazagutía.

Asociacion faunística:

Ammonites: Pachydiscus (Pachydiscus) neurbergicus (Von Hauer).

Inocerámidos: Endocostea aff. impressus (d'Orbigny).

Bioestratigrafía: La especie Pachydiscus (Pachydiscus) neurbergicus es índice de la única zona europea del Maastrichtiende inferior. Lamentablemente, sólo se ha recolectado un ejemplar rodado.

Del ejemplar de Endocostea aff. impressus también se desconoce su posición exacta, por lo que unido a lo incierto de su determinación, no se puede precisar su edad.

\section{MAASTRICHTIENSE SUPERIOR}

Localidades: Andoin y Puerto de Vicuña

Procedencia de la fauna: Colección del MCNA y fauna recolectada por los autores. Se realizó la serie de Pto. de Vicuña (López y Santamaría, 1994).
Litología: Margas arenosas y calizas bioclasticas Fm. del Puerto de Olazagutía.

Asociación faunística:

Ammonites: Anapachydiscus fresvillensis (Seunes), Pachydiscus (Pachydiscus) jacquoti (Seunes), Pachydiscus (P.) sp., Pseudophyllites indra (Forbes), Hoploscaphites constrictus (Sowerby), Baculites anceps Lamarck y Baculites $\mathrm{sp}$.

Otros taxones relevantes: Agerostrea ungulata (Schlotheim), Amphydonte (Amphydonte) pyrenaicum (Leymerie), Exogyra (Exogyra) overwegi (Buch), Neithea (Neithea) striatocostata (Goldfuss), Pholadomya nudolifera Munster, Pycnodonte sp., Rastellum (Rastellum) macropterum Stenzel, Cantharus sp., Neritoma (Neridomus) sp., Otostoma rugosa (Hoeninghaus), Pleurotomaria sp. y Cretirhynchia sp.

Bioestratigrafía: La presencia de A. fresvillensis y $B$. anceps permite datar esta asociación como Maastrichtiense superior (Fig. 4).

Algunas de las especies de bivalvos y gasterópodos recolectadas en el Puerto de Vicuña y Andoin indican una clara edad Maastrichtiense, en concreto su parte superior, este es el caso de Agerostrea ungulata, Rastellum (Rastellum) macropterum y Otostoma rugosa.

\section{CONCLUSIONES}

Los yacimientos de Legutiano/Villareal de Álava presentan una edad Albiense superior, mientras que los de Ullíbarri Gamboa-Embalses de Zadorra abarcan de manera no continua desde la parte baja del Cenomaniense inferior a la parte baja del superior. Sin embargo, en ambos casos parece imprescindible un trabajo de campo que permita precisar las relaciones estratigráficas de estas faunas. La excepcional fauna de inocerámidos de Villareal de Álava, única en Europa, proporcionará información muy valiosa desde los puntos de vista bioestratigráfico, paleobiogeográfico y filogenético.

En el Santoniense no se han obtenido datos que permitan refinar la bioestratigrafía con ammonites. Por su parte, los inocerámidos han permitido confirmar y mejorar la zonación del Santoniense de la Cuenca Navarro-Cántabra.

El Campaniense ha resultado ser el piso que mayor información ha proporcionado. El Campaniense inferior se ha reconocido, si bien la fauna ha resultado ser escasa. En el Campaniense superior se han reconocido tres asociaciones de ammonites, que podrían eventualmente permitir la subdivisión del mismo en tres zonas. Con inocerámidos esto no ha sido posible por el momento.

Los ammonites han permitido reconocer el Maastrichtiense inferior y el superior en la importante localidad de Puerto de Vicuña. El inferior se ha identificado a partir de un ejemplar rodado, mientras que el superior presenta una asociación bastante interesante y que será descrita en futuros trabajos. Por otra parte, algunas especies de bivalvos y gasterópodos permiten confirmar dicha edad.

\section{AGRADECIMIENTOS}

Este trabajo ha sido financiado por el Museo de Ciencias Naturales de Álava. Agradecemos la colaboración, tanto en los trabajos de campo como en el laboratorio de Jesús 
Alonso y de Carmelo Corral. Así como la gentileza de Don Francisco Ignacio Vaz, por habernos permitido el acceso a su colección paleontológica del Albiense superior.

Este trabajo es una contribución al proyecto 362 del IGCP de la UNESCO.

Los autores agradecen al Dr. Miquel Company (Granada) y a un revisor anónimo, sus valiosas sugerencias y críticas, que han ayudado a mejorar el resultado final del trabajo.

\section{BIBLIOGRAFÍA}

Amiot, M. 1982. El Cretácico superior de la Región Navarro-Cántabra. In: El Cretácico de Espana. 88-111. Universidad Complutense. Madrid.

Ciry, R. 1940. Étude géologique d'une partie des provinces de Burgos, Valencia, Léon et Santander. Bull. Soc. Hist. nat. Toulouse, 74, 1-258.

Dhondt. A. V. 1982. Some Spanish Cretaceous bivalves. Cuadernos de Geología ibérica, 8, 847-865.

Floquet, M., Alonso, A. y Meléndez, A. 1982. Cameros-Castilla. El Cretácico superior. In: El Cretácico de Espana. 387-456. Universidad Complutense. Madrid.

Hancock, J. M. 1991. Ammonite scales for the Cretaceous system. Cretaceous Research, 12, 259-291.

Karrenberg, H. 1935. Ammonitenfaunen aus der Nordspanischen Oberkreide. Palaeontographica, 82a, 125-161.

Kauffman, E. G. 1977. Illustrated guide to biostratigraphically important Cretaceous macrofossils, Western Interior Basin, U.S.A. In: Field Guide: North American Paleontological Convention II (Ed. E.G. Kauffman). Mountain Geologists, 14 (3-4), 225-274.
Kennedy, W. J. 1986. Campanian and Maastrichtian ammonites from northern Aquitaine, France. Spec. Pap. Palaeont., 36, 1-145.

Kennedy, W. J. 1987. Ammonites from the type Santonian and adjacent partes of northern Aquitaine, western France. Palaeontology, 30 (4), 765-782, lams. 80-82.

Küchler, T. y Kutz, A. 1989. Bioestratigraphie des Campan bis Unter-Maastrich der E-Barranca und des Urdiroz/ImiscozGebietes (Navarra, N-Spanien). In: Cretaceous of the Western Tethys. Proceedings 3rd international Cretaceous Symposium, Tubingen 1987. (Ed. J. Wiedmann). E. Schweizerbart'sche Verlags-buchhandlung, Stuttgart. 161-190.

Lamolda, M. A. y Martínez, R. 1986. Bioestratigrafía del Coniaciense y Santoniense en el Norte de Burgos-Oeste de Álava. Paleontologia i Evolució, 20, 225-234.

López, G. 1986. Inocerámidos del Cretácico superior de los alrededores de St. Corneli (prov. Lleida). Publicaciones de Geología de la Universitat Autònoma de Barcelona, 22, 1-121.

López, G. 1992a. Paleontología y Bioestratigrafía de los inocerámidos (Bivalvia) del Cretácico superior de la Cuenca Navarro-Cántabra y de la Plataforma Norcastellana. Parte I: Situación geológica de las series realizadas y estudio sistemático de los subgéneros Inoceramus Sowerby y Cremnoceramus Cox. Boletín Geológico y Minero, 103 (2), 210-252.

López, G. 1992b. Paleontología y Bioestratigrafía de los inocerámidos (Bivalvia) del Cretácico superior de la Cuenca Navarro-Cántabra y de la Plataforma Norcastellana. Parte IV: Estudio sistemático del subgénero Cordiceramus Seitz y bioestratigrafía. Boletín Geológico y Minero, 103 (5), 837-892.

López, G. 1993. Aportaciones al conocimiento de la fauna de bivalvos del Cretacico superior de Álava y Navarra. Parte I. Estudios del Museo de Ciencias Naturales de Álava, 8, 5-26.

\section{Lamina I}

1. Pachydiscus launayi (de Grossouvre). Ejemplar n. ${ }^{\circ}$ 1638. Gámiz (Álava). Campaniense inferior.

2. Turrilites scheuzcherianus Bosc. Ejemplar n. ${ }^{\circ} 304$. Embalses del Zadorra (Álava). Campaniense medio.

3. Nostoceras (Bostrychoceras) polyplocum (Roemer). Ejemplar n. ${ }^{\circ}$ 1790. Ullíbarri-Jauregui (Álava). Campaniense superior (Asociación III).

4. Stoliczkaia dispar (d’Orbigny). Ejemplar n. ${ }^{\circ} 2507$ (Colección J.S.). Legutiano. Albiense superior.

5. Hoplitoplacenticeras marroti Coquand. Ejemplar n. ${ }^{\circ}$ 303. Puerto de Vitoria (Álava). Campaniense superior (Asociación I).

6. Pachydiscus haldemsis (Schluter). Ejemplar n. ${ }^{\circ}$ 1633. Puerto de Vitoria (Álava). Campaniense superior (Asociación II).

7. Placenticeras polyopsis (Dujardin). Ejemplar n. ${ }^{\circ} 202$. Bóveda (Álava). Santoniense.

Todos los ejemplares se encuentran depositados en el Museo de Ciencias Naturales de Álava. La escala gráfica corresponde a un centímetro.

\section{Lámina II}

1. Cataceramus dariensis (Dobrov y Pavlova). Ejemplar n. ${ }^{\circ}$ 1754. Ullíbarri-Jauregui (Álava). Campaniense superior (Asociación III).

2. Inoceramus athabaskensis Mac Learn. Ejemplar n. ${ }^{\circ}$ 1643. Villareal de Álava (Álava). Albiense superior.

3. Codiceramus muelleri muelleri (Petrascheck). Ejemplar n. ${ }^{\circ} 1250$. Olazagutia (Navarra). Santoniense superior.

4. Platyceramus undulatoplicatus michaeli (Heinz). Ejemplar n. ${ }^{\circ} 660$. Olazagutia (Navarra). Santoniense inferior.

5. Cataceramus sagensis nebrascensis (Meek). Ejemplar n. ${ }^{\circ}$ 1934. Ullíbarri-Jauregui (Álava). Campaniense superior (Asociación I).

6. Endocostea aff. impressus (d’Orbigny). Ejemplar n. ${ }^{\circ}$ 1757. Puerto de Vicuña (Álava). Maastrichtiense inferior.

Todos los ejemplares se encuentran depositados en el Museo de Ciencias Naturales de Álava. La escala gráfica corresponde a un centímetro. 
Lámina I
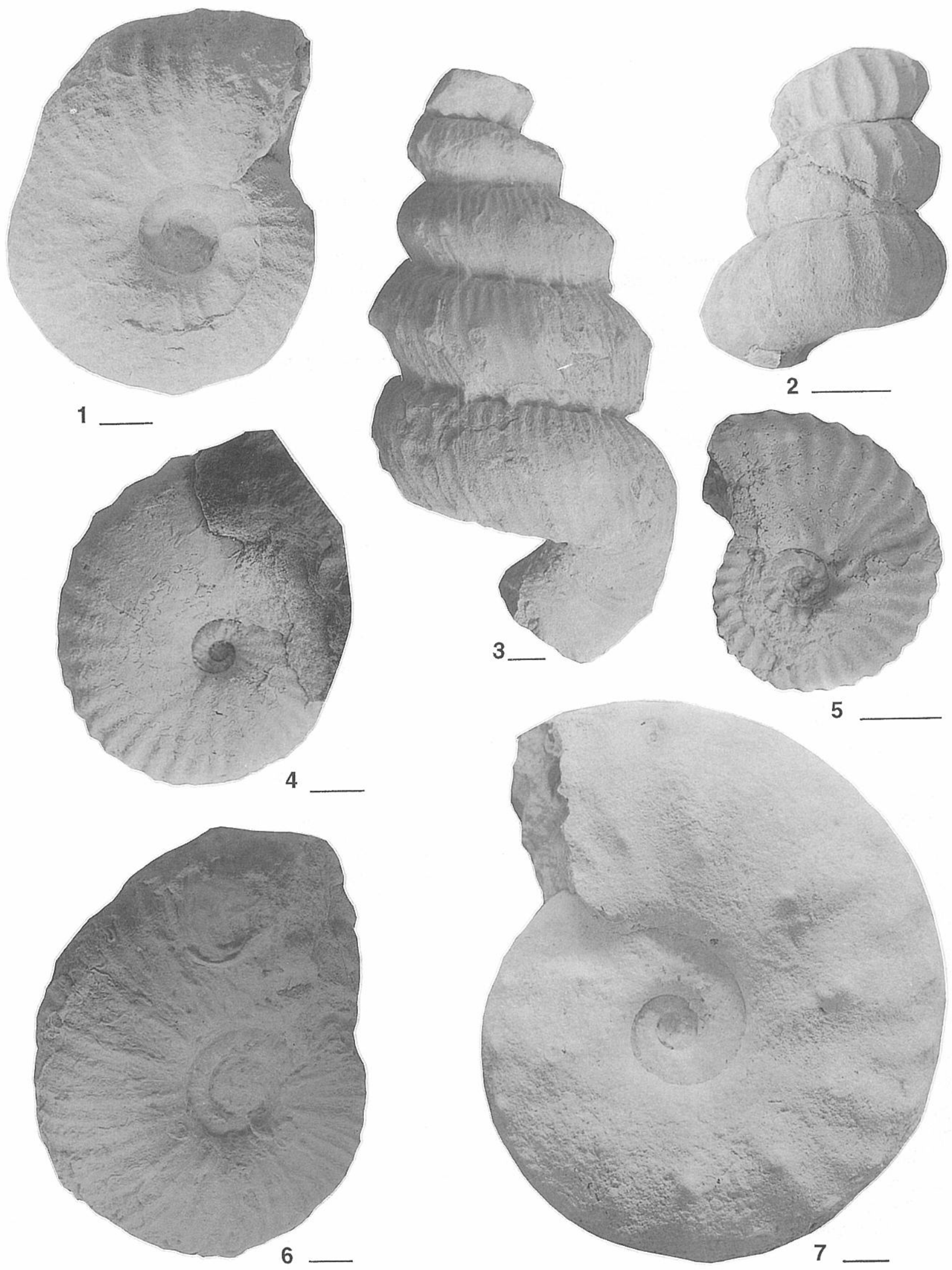
Lámina II
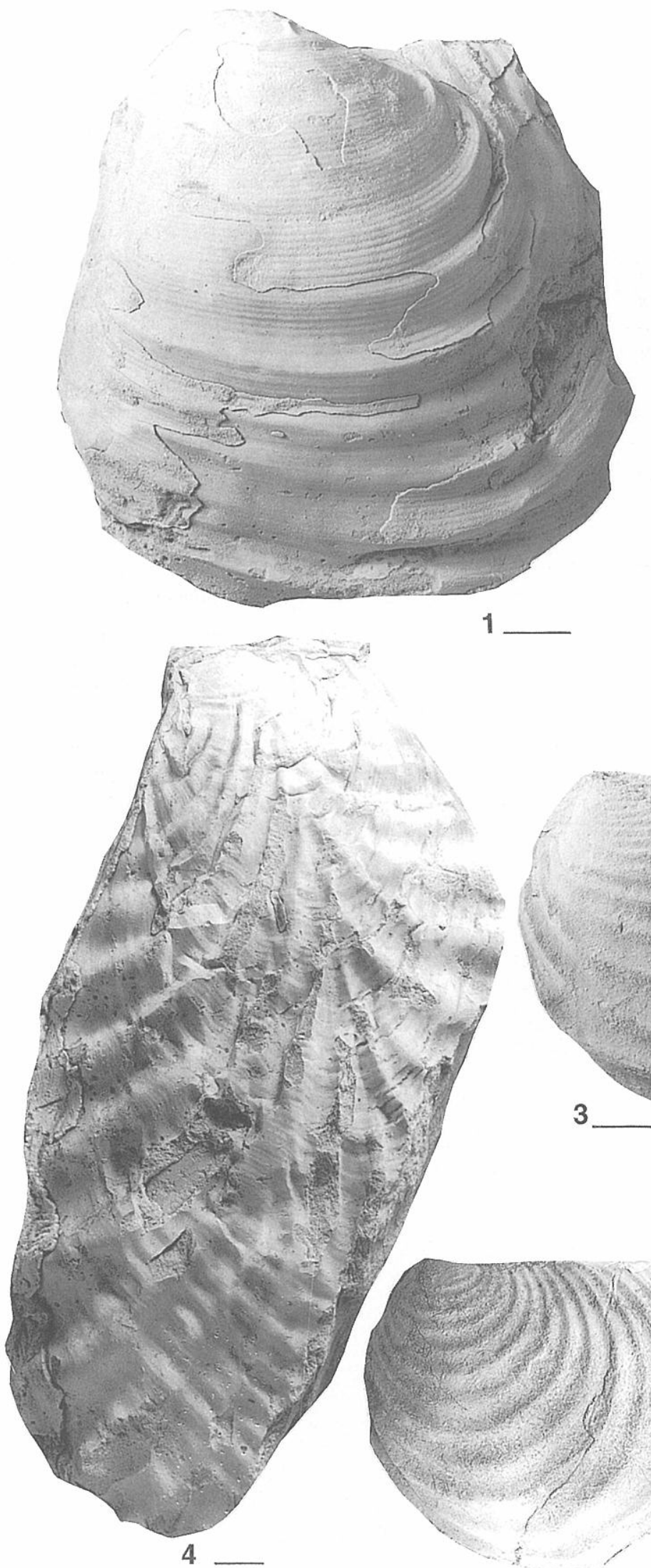

1
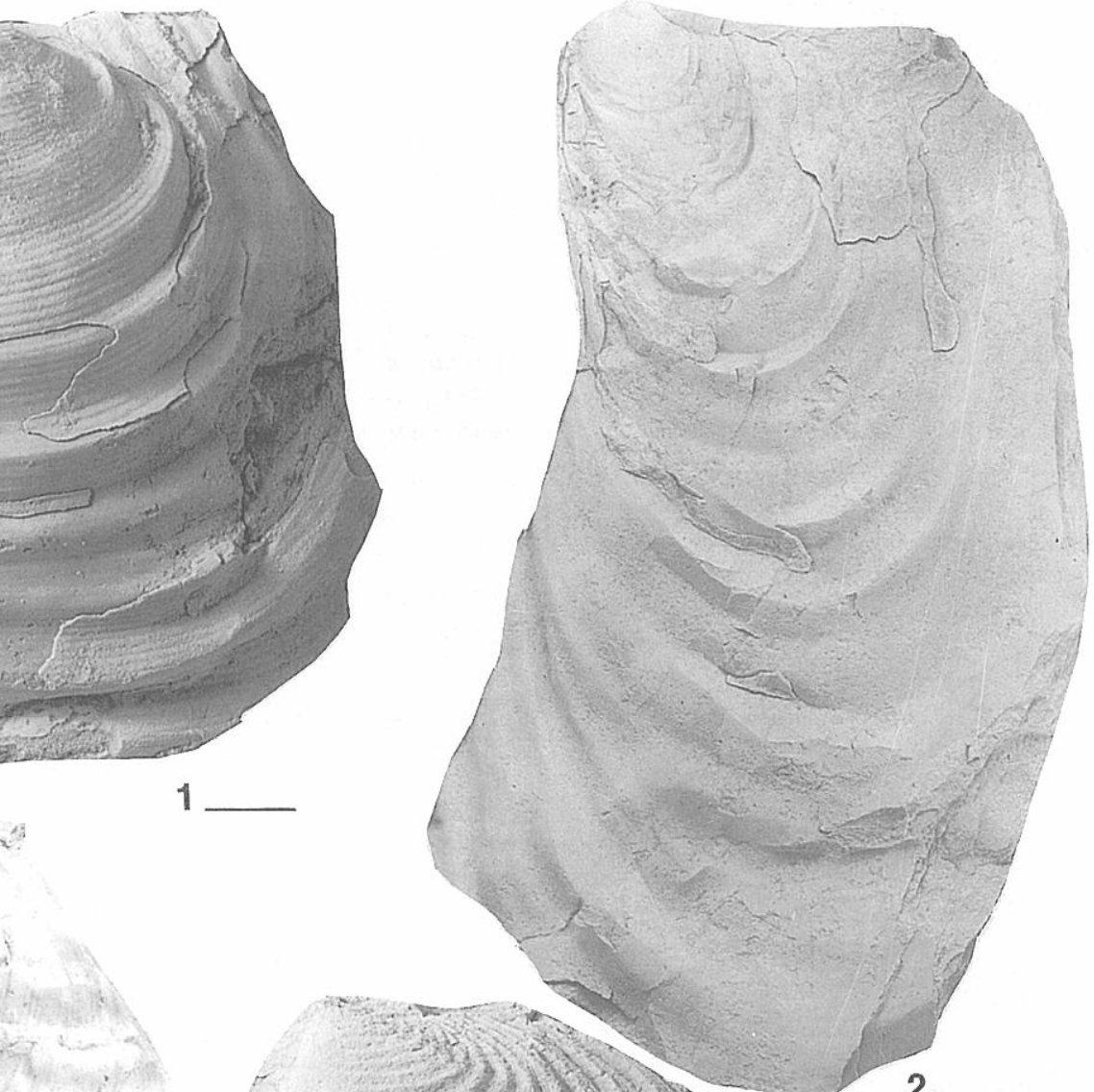

43 की की
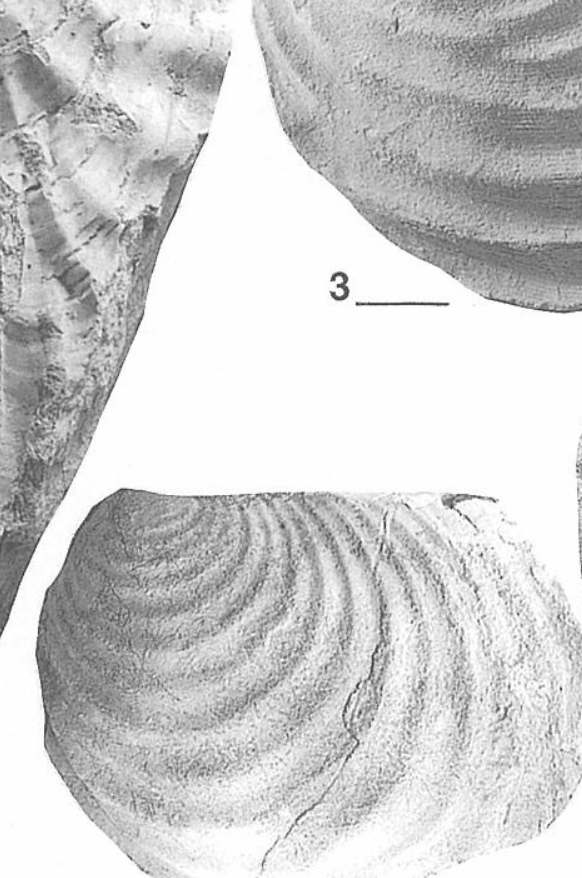

3

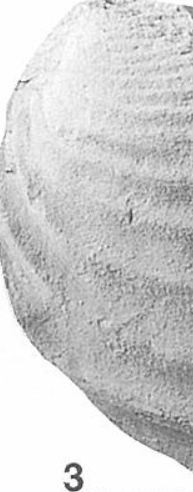

2
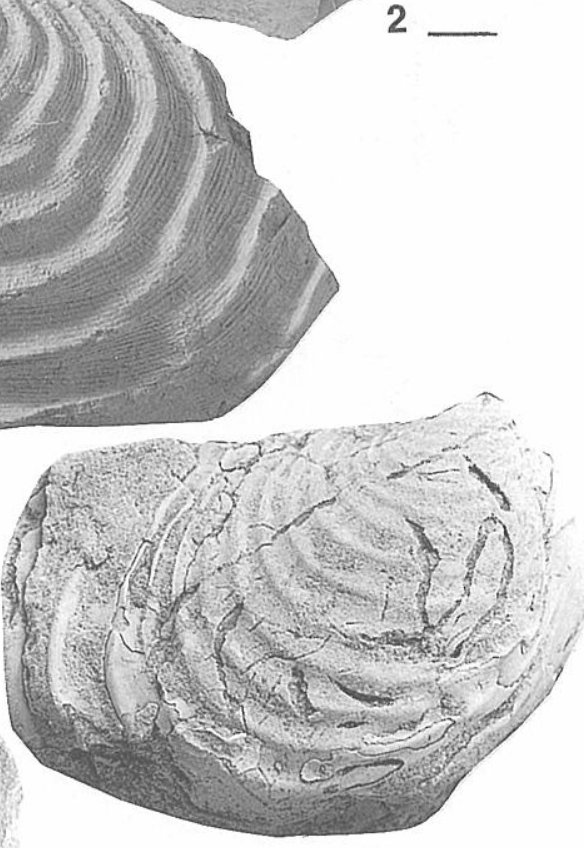

6 
Lopez, G. 1994. Bioestratigrafía de los inocerámidos (Bivalvia) de la Cuenca Navarro-Cántabra y de la Plataforma Norcastellana. Comparación con zonaciones de otras áreas de Europa. Cuadernos de Geología ibérica, 18, 309-336.

López, G. en prensa. Afinidades paleobiogeográficas de los inocerámidos (Bivalvia) del Campaniense y Maastrichtiense de Álava y Navarra. Estudios del Museo de Ciencias Naturales de Álava, $\mathbf{1 0 .}$

López, G. y Santamaría, R. 1992. Correlación entre las zonaciones de ammonites e inocerámidos de parte de la Cuenca Navarro-Cántabra y la Plataforma Norcastellana. III Congreso Geológico de Espana y VIII Congreso Latinoamericano de Geología. Actas, 1, 524-528.

López Sanjaume, G. y Santamaría Zabala, R. 1994. Determinación sistemática de los cefalópodos y pelecípodos del Cretácico alavés, correpondientes a las colecciones del Museo de Ciencias Naturales de Álava, con definición de sus posiciones estratigráficas en la serie local. $58 \mathrm{pp}$. (inédito).

McLearn, F.H. 1943. Trends in some canadian Cretaceous species of Inoceramus. The Canadian Field-Naturalist, 57, 36-46.

Orbigny, A. d'. 1850. Prodrome de Paléontologie stratigraphique universelle des animaux mollusques et rayonnés, 2, $428 \mathrm{pp}$. Masson, París.

Santamaría, R. 1991. Ammonoideos del Cretácico Superior de la plataforma Nord-Castellana y la cuenca Navarro-Cántabra. Paleontología y Bioestratigrafía. Tesis Doctoral Universitat Autònoma de Barcelona, 397 pp., 19 láms. (inédito).

Santamaría, R. 1992. Los Ammonoideos del Cenomaniense superior al Santoniense de la plataforma nord-castellana y la cuenca navarro-cántabra. Parte I. Bioestratigrafía y sistemática: Phylloceratina, Ammonitina (Desmocerataceae y Hoplitaceae) y Ancyloceratina. Treballs del Museu de Geologia de Barcelona, 2, 171-268.

Santamaría Zabala, R. en prensa. Los ammonites del Campaniense de la provincia de Álava. Sistemática y Bioestratigrafía. Estudios del Museo de Ciencias Naturales de Álava, 10.

Seitz, O. 1961. Die Inoceramen des Santon von Nordwestdeutschland. I Teil. Beihgefte zum Geologische Jahrbuch, 46, 3-186, 39 figs.

Seitz, O. 1965. Die Inoceramen des Santon und Unter-Campan von Norwestdeutschland. II Teil. Beihgefte zum Geologische Jahrbuch, 69, 3-194, 11 figs. 26 láms.
Sornay, J. 1978. Inocérames. In: Echelles biostratigraphiques dans le Turonien du Cap Blanc-Nez. (Pas de Calais, F.). (Ed. F. Amedro et al.). Bulletin d'Information des geologes du Bassin de Paris, 15, 3-20.

Sornay, J. 1983. Etude biostratigraphique des faunes d'Inocerames du Senonien francais. Géologié Mediterranée, 10 (3-4), 193-198.

Sornay, J. 1984. Inocerames. In: Synthése géologique du sudest de la France. Crétacé supérieur (Eds. J. Philip et al.). Memoires du Bureau de Recherches Géologiques et Mineres. 125, 343-345.

Tröger, K. A. 1989. Problems of Upper Cretaceous Inoceramid Biostratigraphy and Paleobiogeography in Europe and Western Asia. In: Cretaceous of the Western Tethys. Proceedings 3rd international Cretaceous Symposium, Tubingen 1987. (Ed. J. Wiedmann). E. Schweizerbart'sche Verlagsbuchhandlung, Stuttgart. 911-930.

Wiedmann, J. 1960. Le crétace supérieur de l' Espagne et du Portugal et ses céphalopodes. C. r. Congr. Socs. sav. Dijon, Colloque sur le Crétacé supérieur francais, 1959, 709.764.

Wiedmann, J. 1962. Ammoniten aus der Vascogotischen Kreide (Nordspanien). 1, Phylloceratina, Lytoceratina. Palaeontographica, A118, 119-237.

Wiedmann, J. 1964. Le Crétace supérieur de l'Espagne et du Portugal et ses Céphalopodes. Estudios Geológicos, 20, 107-148.

Wiedmann, J. 1975. Subdivisiones y precisiones bioestratigráficas en el Cretácico superior de las Cadenas celtibéricas. Actas 1er Symposium sobre el Cretácico de la Cordillera Ibérica. Cuenca. 1974, 135-153.

Wiedmann, J. 1979. Mid Cretaceous Events Iberian Field Conference 77. Guide. II Partie. Itineraire geologique à travers le Crétacé Moyen des Chaines vascogotiques et celtibériques (Espagne du Nord). Cuadernos de Geología ibérica, 5, 127-214, 12 láms.

Wiedmann, J. y Kauffman, E. G. 1978. Mid-Cretaceous biostratigraphy of northern Spain. In: Evenements de la partie Moyenne du Crétacé (Eds. R. A. Reyment y G. Thomel). Annales $d u$ Muséum d'Histoire Naturelle de Nice, 4, III.1-III.34, 12 lams.

Manuscrito recibido: 30 de enero, 1995 Manuscrito aceptado: 2 de julio, 1995 